\title{
APPLICATION OF THE FINITE ELEMENT METHOD TO SOLVING THE DUFFING EQUATION OF GROUND MOTION
}

\author{
GODWIN C. E. MBAH, AND KINGSLEY KELECHI IBEH
}

(Received 3 February 2020; Revision Accepted 9 March 2020)

\begin{abstract}
In this paper, we applied the Galerkin Finite Element Method to solve a damped, externally forced, second order ordinary differential equation with cubic nonlinearity known as the Duffing Equation. The Galerkin method uses the functional minimization technique which sets the equation in systems of algebraic equations to be solved. Various simulation on the effect of change on some parametric values of the Duffing equation are shown.
\end{abstract}

KEYWORDS: Galerkin Finite Element Method, stiffness matrix, Duffing Equation, shape functions, basis functions, weight functions.

MS Classifications 2010: 74S05, 65N30, 65M60

\section{INTRODUCTION}

The Finite Element Method (FEM) is a numerical/computational analysis tool used in obtaining approximate solutions to boundary value problems which are governed by a differential equation and a set of boundary conditions. The main idea behind the finite element method is the representation of the domain with smaller subdomains called the finite elements. The distribution of the primary unknown quantity inside an element is then interpolated based on the values at the nodes, so far as nodal elements are used. The interpolation or shape functions must also be a complete set of polynomials. The accuracy of the solution depends, among other factors, on the order of these polynomials, whose order may be linear, quadratic, or higher. The numerical solution corresponds to the values of the primary unknown quantity at the nodes or the edges of the discretized domain. The solution is obtained after solving a system of linear equations. To form such a linear system of equations, the governing differential equation and associated boundary conditions must first be converted to an integro-differential form by using a weighted residual method as the Galerkin approach. This integro-differential formulation is applied to a single element and with the use of proper weight and interpolation functions called the shape functions, the respective element equations are obtained. The assembly of all elements results in a global matrix system that represents the entire domain of the BVP.

The FEM was first used by Clough [1 - 2] and it was invented when aircraft engineers wanted to solve problems relating to structures, wheels and to calculate stress in structures. Since then, the FEM has been used by many researchers in areas such as Electromagnetics [3 - 5], Computational Fluid Dynamics [6], Differential Equations [7], implant dentistry [8], heat transfer [9] and incompressible fluid [10].

\section{THE DUFFING EQUATION}

The Duffing Oscillator or equation is a damped, externally forced, second order nonlinear oscillator with constant coefficients which has gained wide recommendations as the simplest equation which is used to study and describe the chaotic behaviour of a system. It is a nonlinear differential equation which describes an oscillator with a cubic nonlinearity [11]. It was developed by a German Engineer named Georg Wilhem Christian Caspar Duffing in 1918 who aimed to tackle problems of nonlinear oscillators in a systematic way by starting with the linear oscillator and also examining the effects of quadratic and cubic stiffness nonlinearities. He then emphasized the differences between the linear and nonlinear oscillators for both the free and forced vibrations while considering their damping effects.

As a dynamical system that exhibits chaotic behaviour, $\checkmark \quad$ It has a periodic long term behaviour as $t \rightarrow \infty$ making the system to have irregular pattern which does not oscillate nor repeat in a periodic manner. $\checkmark \quad$ It is always vigilant in the change of its initial conditions as any change will alter the trajectory thereby giving a significant difference in its long term behaviour. $\checkmark \quad$ The nonlinearity of the system makes it to exhibit irregular behaviour hence making it to be deterministic.

\footnotetext{
Godwin C. E. Mbah, Department of Mathematics, University of Nigeria, Nsukka, Nigeria.

Kingsley Kelechi Ibeh, Department of Mathematics, University of Nigeria, Nsukka, Nigeria.
} 
The general Duffing equation is given as

$\ddot{x}+\delta \dot{x} \pm \alpha x+\beta x^{3}=\gamma \cos (\omega t)$

where the unknown function $x=x(t)$ is the displacement at time $t$. The damping factor $\delta$ controls the size of the damping. The $\alpha$ controls the size of the stiffness and the $\beta$ controls the amount of nonlinearity in the restoring force. If $\beta=0$, the Duffing equation describes a damped and driven simple harmonic oscillator. The quantity $\gamma$ controls the amplitude of the periodic driving force. If $\gamma=0$, we have a system without driving force. The quantity $\omega$ controls the frequency of the periodic driving force. See [12 - 14]. When the Duffing equation has a negative linear stiffness, it is said to be a double-well potential. See [15 - 17]. The Duffing double-well potential equation is given as

$\ddot{x}+\delta \dot{x}-\alpha x+\beta x^{3}=\gamma \cos (\omega t)$

THE FINITE ELEMENT METHOD

The Finite Element Method is an element wise application of the weighted residual or Galerkin method which involves the following steps:

a. Discretization of the domain

b. Formulation or derivation of element equations

c. Assembly of the element equations

d. Imposition of the boundary conditions

e. Solution of the assembled equations. See $[18,19]$

\section{APPLICATION:}

Consider the given Duffing oscillator, $\ddot{\mathrm{x}}+\delta \dot{\mathrm{x}}+\alpha \mathrm{x}+\beta \mathrm{x}^{3}=\gamma \cos w \mathrm{t}$

Subject to $\mathrm{x}(0)=0, \dot{\mathrm{x}}(1)=1$

We seek for an approximate solution of the form

$X(t)=\sum_{i=1}^{n} \alpha_{i} \xi_{i}$

The error or residue $E$ is given as

$$
\mathrm{E}=\ddot{\mathrm{x}}+\delta \dot{\mathrm{x}}+\alpha \mathrm{x}+\beta \mathrm{x}^{3}-\gamma \cos \omega \mathrm{t}
$$

and by Galerkin FEM,

$$
\int_{0}^{l} E w(t) d t=0
$$

\section{Step 1: Discretization of the Domain}

Let the function be divided into four elements in the domain $[0,1]$. Let $w=w(t)$ be the test function and let $s_{1}$ ands $s_{2}$ be successive node points.

For each element $\mathrm{e}_{\mathrm{i}}, \mathrm{i}=1,2,3,4$, we have two nodes and from (

$\int_{s_{1}}^{s_{2}}\left(\ddot{x}+\delta \dot{x}+\alpha x+\beta x^{3}\right) w d t-\int_{s_{1}}^{s_{2}} \gamma w \cos \omega d t=0$

Step 2: Derivation of element equations

$$
\Rightarrow \int_{s_{1}}^{s_{2}}\left(-\dot{x} \dot{w}+\delta \dot{x} w+\alpha x w+\beta x^{3} w\right) d t=\int_{s_{1}}^{s_{2}} \gamma w \cos \omega t d t-\left.w \dot{x}\right|_{s_{1}} ^{s_{2}}
$$

Let

$$
w(t)=\sum_{j=1}^{2} \gamma_{j} \psi_{j}(t) \text { and } x(t)=\sum_{i=1}^{2} \alpha_{i} \psi_{i}(t)
$$

where $\psi_{i}$ and $\psi_{j}$ are shape functions; $\gamma_{i}$ and $\alpha_{i}$ are coefficients for the particular node which is 1 and 0 at other nodes.

$$
\psi_{\mathrm{i}}=\frac{\mathrm{s}_{\mathrm{i}+1}-\mathrm{t}}{\mathrm{s}_{\mathrm{i}+1}-s_{\mathrm{i}}}, \quad \psi_{\mathrm{i}+1}=\frac{\mathrm{t}-\mathrm{s}_{\mathrm{i}}}{\mathrm{s}_{\mathrm{i}+1}-s_{\mathrm{i}}}
$$

But $\mathrm{s}_{1}=\mathrm{s}_{\mathrm{i}}, \mathrm{s}_{2}=\mathrm{s}_{\mathrm{i}+1}$, wheni $=1$ andh $=\mathrm{s}_{\mathrm{i}+1}-\mathrm{s}_{\mathrm{i}}$

Substituting $(7,8)$ into $(6)$, we obtain

$\int_{s_{i}}^{s_{i+1}}\left(-\dot{\psi}_{1} \dot{\psi}_{j}+\delta \dot{\psi}_{1} \psi_{\mathrm{j}}+\alpha \psi_{\mathrm{i}} \psi_{\mathrm{j}}+\beta \psi_{\mathrm{i}}^{3} \psi_{\mathrm{j}}\right) \mathrm{dt}=\gamma \int_{\mathrm{s}_{1}}^{s_{2}} \psi_{\mathrm{j}} \cos \omega t \mathrm{tdt}-\left.\psi_{\mathrm{j}} \dot{\mathrm{x}}\right|_{\mathrm{s}_{1}} ^{s_{2}}$

Equation (9) can be written as

$$
\mathrm{k}_{\mathrm{ij}}^{\mathrm{e}}=\mathrm{g}_{\mathrm{i}}^{\mathrm{e}}
$$


where $\mathrm{k}_{\mathrm{ij}}^{\mathrm{e}}$ is the stiffness matrix and it is given as

$\mathrm{k}_{\mathrm{ij}}^{\mathrm{e}}=\int_{\mathrm{s}_{\mathrm{i}}}^{\mathrm{s}_{\mathrm{i}+1}}\left(-\dot{\psi}_{1} \dot{\psi}_{\mathrm{j}}+\delta \dot{\psi}_{1} \psi_{\mathrm{j}}+\alpha \psi_{\mathrm{i}} \psi_{\mathrm{j}}+\beta \psi_{\mathrm{i}}^{3} \psi_{\mathrm{j}}\right) \mathrm{dt}$

$\mathrm{g}_{\mathrm{i}}^{\mathrm{e}}=\gamma \int_{\mathrm{s}_{1}}^{\mathrm{s}_{2}} \psi_{\mathrm{j}} \cos \omega \mathrm{tdt}-\left.\psi_{\mathrm{j}}\right|_{\mathrm{s}_{1}} ^{\mathrm{s}_{2}}$

Then, from (11)

and

$$
\mathrm{k}_{11}=\alpha \frac{\mathrm{h}}{3}+\frac{\beta \mathrm{h}}{5}-\frac{1}{\mathrm{~h}}-\frac{\delta}{2}=\mathrm{k}_{22}
$$

$$
k_{12}=\left\{\frac{1}{h}-\frac{\delta}{2}-\frac{\alpha}{2 h}\left(s_{2}+2 s_{1}\right)\left(s_{2}+s_{1}\right)+\frac{\beta h}{20}\right\}=k_{21}
$$

Hence, for a typical element, its stiffness matrix is

$$
K^{e}=\left[\begin{array}{ll}
k_{11} & k_{12} \\
k_{21} & k_{22}
\end{array}\right]
$$

\section{Step 3: Assembling of element equations}

For every element,

$$
K^{e}=\left[\begin{array}{cc}
\frac{\alpha h}{3}+\frac{\beta h}{5}-\frac{1}{h}-\frac{\delta}{2} & \frac{1}{h}-\frac{\delta}{2}-\frac{\alpha}{2 h}\left(s_{2}+2 s_{1}\right)\left(s_{2}+s_{1}\right)+\frac{\beta h}{20} \\
\frac{1}{h}-\frac{\delta}{2}-\frac{\alpha}{2 h}\left(s_{2}+2 s_{1}\right)\left(s_{2}+s_{1}\right)+\frac{\beta h}{20} & \frac{\alpha h}{3}+\frac{\beta h}{5}-\frac{1}{h}-\frac{\delta}{2}
\end{array}\right]
$$

The subscripts 1 and 2 are nodes 1 and 2 of a particular element. When we start assembling the matrices, the subscripts will be relabeled to take note of the element in question.

Then

$$
\begin{gathered}
K^{1}=\left(\begin{array}{ll}
\frac{\alpha}{12}+\frac{\beta}{20}-\frac{\delta}{2}-4 & \frac{\beta}{80}-\frac{\delta}{2}-\frac{\alpha}{8}+4 \\
\frac{\beta}{80}-\frac{\delta}{2}-\frac{\alpha}{8}+4 & \frac{\alpha}{12}+\frac{\beta}{20}-\frac{\delta}{2}-4
\end{array}\right), \quad K^{2}=\left(\begin{array}{cc}
\frac{\alpha}{12}+\frac{\beta}{20}-\frac{\delta}{2}-4 & \frac{\beta}{80}-\frac{\delta}{2}-\frac{3}{2} \alpha+4 \\
\frac{\beta}{80}-\frac{\delta}{2}-\frac{3}{2} \alpha+4 & \frac{\alpha}{12}+\frac{\beta}{20}-\frac{\delta}{2}-4
\end{array}\right) \\
K^{3}=\left(\begin{array}{ll}
\frac{\alpha}{12}+\frac{\beta}{20}-\frac{\delta}{2}-4 & \frac{\beta}{80}-\frac{\delta}{2}-\frac{3}{2} \alpha+4 \\
\frac{\beta}{80}-\frac{\delta}{2}-\frac{3}{2} \alpha+4 & \frac{\alpha}{12}+\frac{\beta}{20}-\frac{\delta}{2}-4
\end{array}\right), K^{4}=\left(\begin{array}{cc}
\frac{\alpha}{12}+\frac{\beta}{20}-\frac{\delta}{2}-4 & \frac{\beta}{80}-\frac{\delta}{2}-\frac{35}{4} \alpha+4 \\
\frac{\beta}{80}-\frac{\delta}{2}-\frac{35}{4} \alpha+4 & \frac{\alpha}{12}+\frac{\beta}{20}-\frac{\delta}{2}-4
\end{array}\right)
\end{gathered}
$$

The global system of matrices equals:

$$
K=\left(\begin{array}{ccccc}
k_{11}^{1} & k_{12}^{1} & 0 & 0 & 0 \\
k_{21}^{1} & k_{22}^{1}+k_{11}^{2} & k_{12}^{2} & 0 & 0 \\
0 & k_{21}^{2} & k_{22}^{2}+k_{11}^{3} & k_{12}^{3} & 0 \\
0 & 0 & k_{21}^{3} & k_{22}^{3}+k_{11}^{4} & k_{12}^{4} \\
0 & 0 & 0 & k_{21}^{4} & k_{22}^{4}
\end{array}\right)
$$


which gives to

$$
\begin{aligned}
& \left(\frac{\alpha}{12}+\frac{\beta}{20}-\frac{\delta}{2}-4 \quad \frac{\beta}{80}-\frac{\delta}{2}-\frac{\alpha}{8}+4\right. \\
& \frac{\beta}{80}-\frac{\delta}{2}-\frac{\alpha}{8}+4 \quad \frac{\alpha}{6}+\frac{\beta}{10}-\delta-2 \quad \frac{\beta}{80}-\frac{\delta}{2}-\frac{3}{2} \alpha+4 \quad 0 \quad 0 \\
& K=\quad 0 \quad \frac{\beta}{80}-\frac{\delta}{2}-\frac{3}{2} \alpha+4 \quad \frac{\alpha}{6}+\frac{\beta}{10}-\delta-2 \quad \frac{\beta}{80}-\frac{\delta}{2}-\frac{35}{8} \alpha+4 \quad 0 \\
& 0 \quad 0 \quad \frac{\beta}{80}-\frac{\delta}{2}-\frac{35}{8} \alpha+4 \quad \frac{\alpha}{6}+\frac{\beta}{10}-\delta-2 \quad \frac{\beta}{80}-\frac{\delta}{2}-\frac{35}{4} \alpha+4 \\
& 0 \quad 0 \quad 0 \quad \frac{\beta}{80}-\frac{\delta}{2}-\frac{35}{4} \alpha+4 \quad \frac{\alpha}{12}+\frac{\beta}{20}-\frac{\delta}{2}-4
\end{aligned}
$$

The load vectors are given as (12)

Then each of the load vectors gives:

$$
G_{i}=\left[\begin{array}{c}
\frac{\gamma}{\omega h}\left[s_{1} \sin \omega h-\frac{\cos \omega h}{\omega}\right] \\
\left.\frac{\gamma}{h}\left[\left(t-s_{1}\right) \frac{\sin \omega t}{\omega}+\frac{\cos \omega t}{w^{2}}\right]\right|_{s_{1}} ^{s_{2}}
\end{array}\right]
$$

The global load vectors are summarized as thus:

$$
G=\left(\begin{array}{l}
g_{1}^{1}-x(0) \\
g_{2}^{1}+g_{1}^{2} \\
g_{2}^{2}+g_{1}^{3} \\
g_{2}^{3}+g_{1}^{4} \\
g_{2}^{4}+\dot{x}(1)
\end{array}\right)
$$

which gives

$$
G=\left(\begin{array}{c}
-\frac{4 \gamma}{\omega} \cos \frac{\omega}{4}-x(0) \\
\frac{2 \gamma}{\omega} \sin \frac{\omega}{4} \\
\frac{2 \gamma}{\omega} \sin \frac{\omega}{4} \\
\frac{2 \gamma}{\omega} \sin \frac{\omega}{4} \\
\frac{4 \gamma}{\omega}\left(\cos \frac{\omega}{4}-\frac{1}{2} \sin \frac{\omega}{4}\right)+\dot{x}(1)
\end{array}\right)
$$

Then combining the equations together, we have

$$
\left(\begin{array}{ccccc}
\frac{\alpha}{12}+\frac{\beta}{20}-\frac{\delta}{2}-4 & \frac{\beta}{80}-\frac{\delta}{2}-\frac{\alpha}{8}+4 & 0 & 0 & 0 \\
\frac{\beta}{80}-\frac{\delta}{2}-\frac{\alpha}{8}+4 & \frac{\alpha}{6}+\frac{\beta}{10}-\delta-2 & \frac{\beta}{80}-\frac{\delta}{2}-\frac{3}{2} \alpha+4 & 0 & 0 \\
0 & \frac{\beta}{80}-\frac{\delta}{2}-\frac{3}{2} \alpha+4 & \frac{\alpha}{6}+\frac{\beta}{10}-\delta-2 & \frac{\beta}{80}-\frac{\delta}{2}-\frac{35}{8} \alpha+4 & 0 \\
0 & 0 & \frac{\beta}{80}-\frac{\delta}{2}-\frac{35}{8} \alpha+4 & \frac{\alpha}{6}+\frac{\beta}{10}-\delta-2 & \frac{\beta}{80}-\frac{\delta}{2}-\frac{35}{4} \alpha+4 \\
0 & 0 & 0 & \frac{\beta}{80}-\frac{\delta}{2}-\frac{35}{4} \alpha+4 & \frac{\alpha}{12}+\frac{\beta}{20}-\frac{\delta}{2}-4
\end{array}\right)=\left(\begin{array}{c}
u_{1} \\
u_{2} \\
u_{3} \\
u_{4} \\
u_{5}
\end{array}\right)\left(\begin{array}{c}
-\frac{4 \gamma}{\omega} \cos \frac{\omega}{4}-x(0) \\
\frac{2 \gamma}{\omega} \sin \frac{\omega}{4} \\
\frac{2 \gamma}{\omega} \sin \frac{\omega}{4} \\
\frac{2 \gamma}{\omega} \sin \frac{\omega}{4} \\
\frac{4 \gamma}{\omega}\left(\cos \frac{\omega}{4}-\frac{1}{2} \sin \frac{\omega}{4}\right)+\dot{x}(1)
\end{array}\right)
$$


The first and last rows have been covered by the boundary conditions and hence

$$
\left(\begin{array}{ccc}
\frac{\alpha}{6}+\frac{\beta}{10}-\delta-2 & \frac{\beta}{80}-\frac{\delta}{2}-\frac{3}{2} \alpha+4 & 0 \\
\frac{\beta}{80}-\frac{\delta}{2}-\frac{3}{2} \alpha+4 & \frac{\alpha}{6}+\frac{\beta}{10}-\delta-2 & \frac{\beta}{80}-\frac{\delta}{2}-\frac{35}{8} \alpha+4 \\
0 & \frac{\beta}{80}-\frac{\delta}{2}-\frac{35}{8} \alpha+4 & \frac{\alpha}{6}+\frac{\beta}{10}-\delta-2
\end{array}\right)\left(\begin{array}{l}
u_{2} \\
u_{3} \\
u_{4}
\end{array}\right)=\left(\begin{array}{l}
\frac{2 \gamma}{\omega} \sin \frac{\omega}{4} \\
\frac{2 \gamma}{\omega} \sin \frac{\omega}{4} \\
\frac{2 \gamma}{\omega} \sin \frac{\omega}{4}
\end{array}\right)
$$

Then we solve for $u_{2}, u_{3}, u_{4}$ which gives $\alpha_{2}, \alpha_{3}, \alpha_{4}$.

$$
\left(\begin{array}{l}
u_{2} \\
u_{3} \\
u_{4}
\end{array}\right)=\left(\begin{array}{ccc}
\frac{\alpha}{6}+\frac{\beta}{10}-\delta-2 & \frac{\beta}{80}-\frac{\delta}{2}-\frac{3}{2} \alpha+4 & 0 \\
\frac{\beta}{80}-\frac{\delta}{2}-\frac{3}{2} \alpha+4 & \frac{\alpha}{6}+\frac{\beta}{10}-\delta-2 & \frac{\beta}{80}-\frac{\delta}{2}-\frac{35}{8} \alpha+4 \\
0 & \frac{\beta}{80}-\frac{\delta}{2}-\frac{35}{8} \alpha+4 & \frac{\alpha}{6}+\frac{\beta}{10}-\delta-2
\end{array}\right)^{-1}\left(\begin{array}{c}
\frac{2 \gamma}{\omega} \sin \frac{\omega}{4} \\
\frac{2 \gamma}{\omega} \sin \frac{\omega}{4} \\
\frac{2 \gamma}{\omega} \sin \frac{\omega}{4}
\end{array}\right)
$$

Therefore,

$$
\begin{aligned}
& u_{2}=\frac{\frac{2 \gamma}{\omega} \sin \frac{\omega}{4}\left[\left(\frac{\alpha}{6}+\frac{\beta}{10}-\delta-2\right)^{2}+\frac{23}{8} \alpha\left(\frac{\beta}{80}-\frac{\delta}{2}-\frac{35}{8} \alpha+4\right)\right]}{\left(\frac{\alpha}{6}+\frac{\beta}{10}-\delta-2\right)\left[\left(\frac{\alpha}{6}+\frac{\beta}{10}-\delta-2\right)^{2}-\left(\frac{\beta}{80}-\frac{\delta}{2}-\frac{3}{2} \alpha+4\right)^{2}-\left(\frac{\beta}{80}-\frac{\delta}{2}-\frac{35}{8} \alpha+4\right)^{2}\right]}=\alpha_{2} \\
& u_{3}=\frac{\frac{2 \gamma}{\omega} \sin \frac{\omega}{4}\left[\frac{145}{24} \alpha+\frac{3}{40} \beta-10\right]}{\left(\frac{\alpha}{6}+\frac{\beta}{10}-\delta-2\right)\left[\left(\frac{\alpha}{6}+\frac{\beta}{10}-\delta-2\right)^{2}-\left(\frac{\beta}{80}-\frac{\delta}{2}-\frac{3}{2} \alpha+4\right)^{2}-\left(\frac{\beta}{80}-\frac{\delta}{2}-\frac{35}{8} \alpha+4\right)^{2}\right]}=\alpha_{3} \\
& u_{4}=\frac{\frac{2 \gamma}{\omega} \sin \frac{\omega}{4}\left[\left(\frac{\alpha}{6}+\frac{\beta}{10}-\delta-2\right)^{2}-\left(\frac{\beta}{80}-\frac{\delta}{2}-\frac{3}{2} \alpha+4\right)^{2}-\left(\frac{\beta}{80}-\frac{\delta}{2}-\frac{35}{8} \alpha+4\right)\left(\frac{\beta}{80}-\frac{\delta}{2}-\frac{3}{2} \alpha+5\right)\right]}{\left(\frac{\alpha}{6}+\frac{\beta}{10}-\delta-2\right)\left[\left(\frac{\alpha}{6}+\frac{\beta}{10}-\delta-2\right)^{2}-\left(\frac{\beta}{80}-\frac{\delta}{2}-\frac{3}{2} \alpha+4\right)^{2}-\left(\frac{\beta}{80}-\frac{\delta}{2}-\frac{35}{8} \alpha+4\right)^{2}\right]}=\alpha_{4}
\end{aligned}
$$

The general weak solution of the Duffing equation using FEM is given as:

$$
\begin{gathered}
X(t)=\xi_{1}+\frac{\frac{2 \gamma}{\omega} \sin \frac{\omega}{4}\left[\left(\frac{\alpha}{6}+\frac{\beta}{10}-\delta-2\right)^{2}+\frac{23}{8} \alpha\left(\frac{\beta}{80}-\frac{\delta}{2}-\frac{35}{8} \alpha+4\right)\right]}{\left(\frac{\alpha}{6}+\frac{\beta}{10}-\delta-2\right)\left[\left(\frac{\alpha}{6}+\frac{\beta}{10}-\delta-2\right)^{2}-\left(\frac{\beta}{80}-\frac{\delta}{2}-\frac{3}{2} \alpha+4\right)^{2}-\left(\frac{\beta}{80}-\frac{\delta}{2}-\frac{35}{8} \alpha+4\right)^{2}\right]_{2}} \\
+\frac{\frac{2 \gamma}{\omega} \sin \frac{\omega}{4}\left[\frac{145}{24} \alpha+\frac{3}{40} \beta-10\right]}{\left(\frac{\alpha}{6}+\frac{\beta}{10}-\delta-2\right)\left[\left(\frac{\alpha}{6}+\frac{\beta}{10}-\delta-2\right)^{2}-\left(\frac{\beta}{80}-\frac{\delta}{2}-\frac{3}{2} \alpha+4\right)^{2}-\left(\frac{\beta}{80}-\frac{\delta}{2}-\frac{35}{8} \alpha+4\right)^{2}\right]_{3}} \\
+\frac{\frac{2 \gamma}{\omega} \sin \frac{\omega}{4}\left[\left(\frac{\alpha}{6}+\frac{\beta}{10}-\delta-2\right)^{2}-\left(\frac{\beta}{80}-\frac{\delta}{2}-\frac{3}{2} \alpha+4\right)^{2}-\left(\frac{\beta}{80}-\frac{\delta}{2}-\frac{3}{2} \alpha+5\right)\left(\frac{\beta}{80}-\frac{\delta}{2}-\frac{35}{8} \alpha+4\right)\right]}{\left(\frac{\alpha}{6}+\frac{\beta}{10}-\delta-2\right)\left[\left(\frac{\alpha}{6}+\frac{\beta}{10}-\delta-2\right)^{2}-\left(\frac{\beta}{80}-\frac{\delta}{2}-\frac{3}{2} \alpha+4\right)^{2}-\left(\frac{\beta}{80}-\frac{\delta}{2}-\frac{35}{8} \alpha+4\right)^{2}\right]} \xi_{4} \xi_{5}
\end{gathered}
$$

where

$$
\xi_{1}=x(0)=0, \quad \xi_{5}=\dot{x}(1)=l
$$

are the boundary conditions.

Equation (18) is the finite element method solution of the Duffing equation. To obtain the numerical strings of approximate solutions, we give numerical values to the constants $\alpha, \beta, \gamma, \delta$, and $\omega$. 


\section{NUMERICAL EXPERIMENTS}

In this section, we would give some numerical values to the parameters of the Duffing equation whose solution was derived in (18) above. These approximating values will make the solution be in form of a polynomial. We take two examples.

a. Let $\alpha=1, \beta=5, \delta=0.02, \gamma=8$ and $\omega=0.5$ then, the solution (18) becomes

$$
X(t)=\xi_{1}+0.28079 \xi_{2}-0.03861 \xi_{3}-0.03812 \xi_{4}+\xi_{5}
$$

b. Let $\alpha=-2, \delta=0.1, \beta=2, \gamma=3$ and $\omega=1.2$, then, the solution (18) becomes

$$
X(t)=\xi_{1}-0.00389 \xi_{2}-0.00125 \xi_{3}-0.00832 \xi_{4}+\xi_{5}
$$

\section{SOME NUMERICAL SIMULATIONS AND INTERPRETATIONS}

We show the numerical simulations of the behaviour of the damped Duffing oscillator which was obtained by the use of the Galerkin finite element techniques. Using MATLAB ODE 45 package, we show in figures below, the effects of the change in damping factor $\delta$, and the amount of nonlinearity $\beta$ even at steady state and variation in size of stiffness $\alpha$.
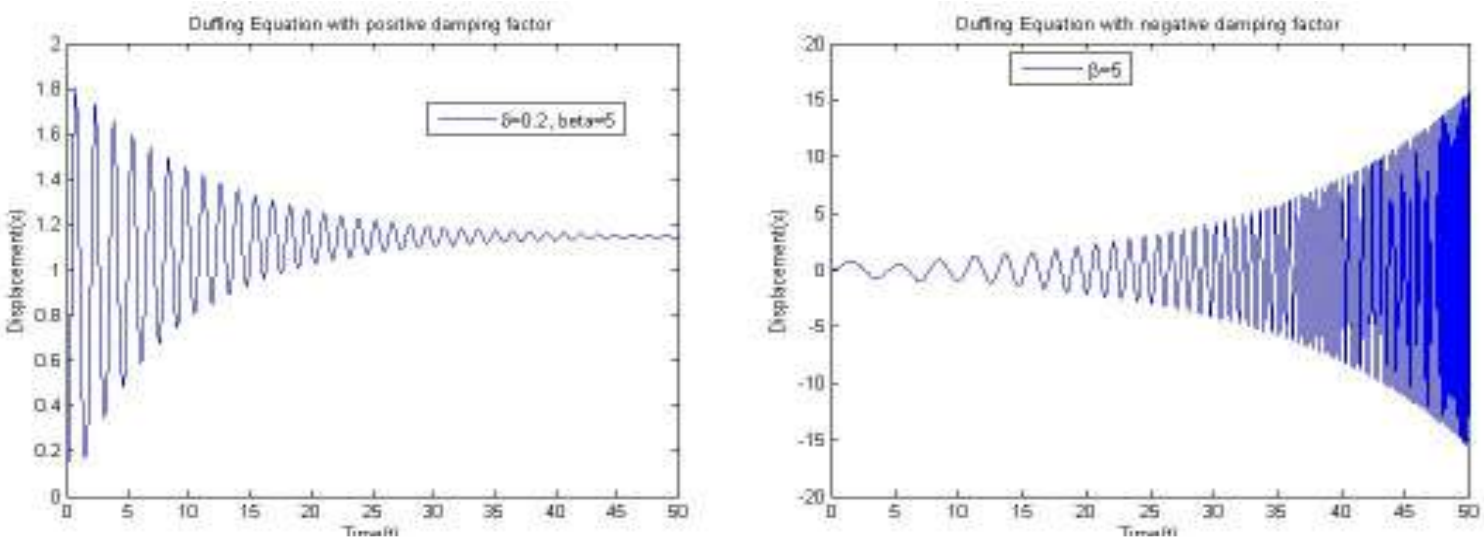

Fig. A and B: Duffing Oscillator with positive and negative damping factor. The damping has effects on earthquake occurrence as the positivity helps to reduce the tremor. Negative damping factor increases its chaotic behaviour.
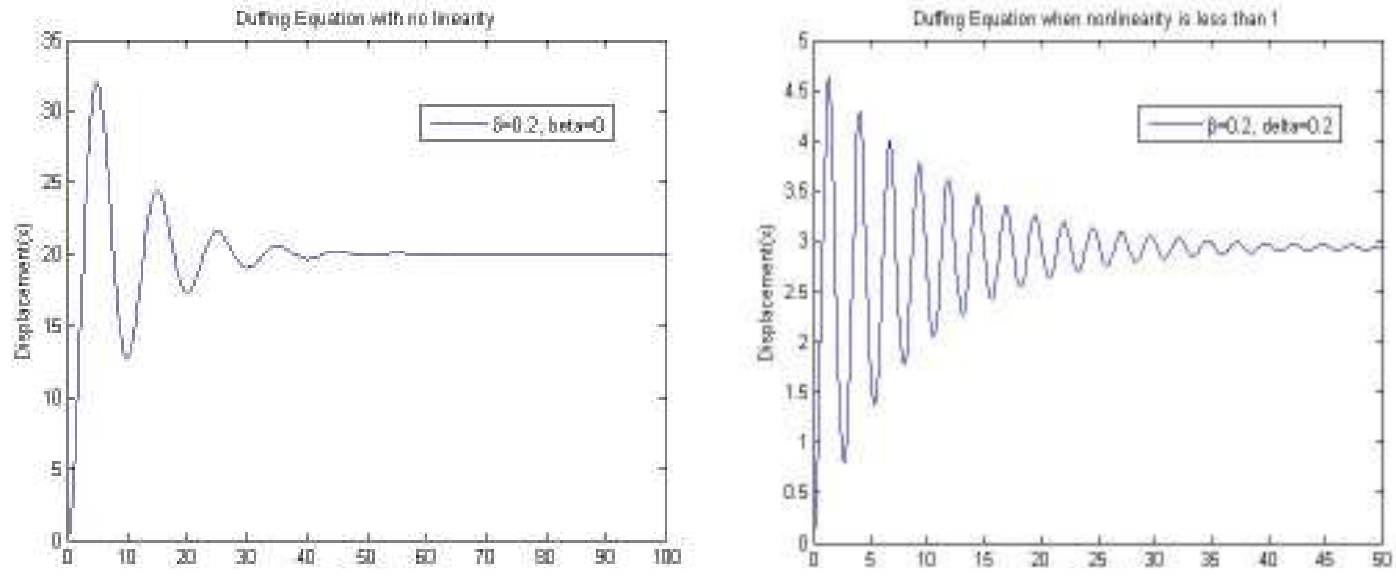

Fig. C and D: Duffing Oscillator with no linear term and when linearity is less than 1. 

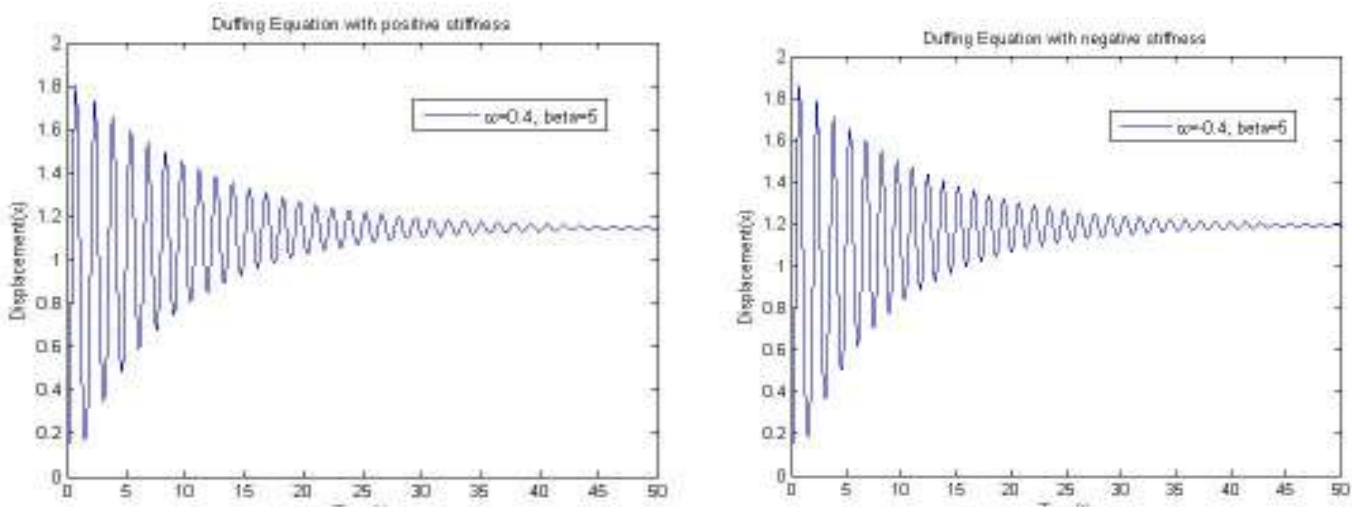

Fig. E and F: Duffing Oscillator with positive and negative stiffness gives no change.

\section{CONCLUSION}

In this research, we have applied the use of the Finite Element Method to solving the Duffing Equation of ground motion. The FEM has been seen as a powerful tool for solving nonlinear dynamic problems which have no definite analytical solution. This method gave us a promising iterative result that such equation can be reduced to a polynomial.

\section{REFERENCES}

R. N. Clough: "The Finite Element Method in Plane Stress Analysis", Proceedings of the Second ASCE Conference on Electronic Computations; Pittsburgh, PA, September 1960.

R. N. Clough: "Early History of the Finite Element Method from the view point of a Pioneer". Int. Journal of Numerical Methods Eng. 60, 2004. 287.

A. C. Polycarpou: "Introduction to the Finite Element Method in Electromagnetics", $1^{\text {st }}$ Edition. Morgan and Claypool Publishers, USA, 2006.

J. Jin: "The Finite Element Method in Electromagnetics", $2^{\text {nd }}$ Ed., Wiley Pub. Canada. 2002.

J. Jin, D. J. Riley: "Finite Element Analysis of Antennas and Arrays", John Wiley and Publishers, IEEE Press, Canada. 2009.

J. Iannelli: "Characteristic Finite Element Methods in Computational Fluid Dynamics", Computational Fluid and Solid Mechanics Book Series, Springer Publishers, Germany, 2006.

G. Evans et al: "Numerical Methods for Partial Differential Equations", Springer Undergraduate Mathematics Series, Springer - Verlag, London., 2000.

J. P. Geng et al: "Application of Finite Element Analysis in Implant Dentistry; A Review of the Literature"; Journal of Prosthetic Dentistry, 2001.

P. Hemmingway, A. Tolooiyan: "Numerical and Finite Element Analysis of Heat Transfer in a Closed Loop Geothermal System", Int'I Journal of Green Energy - Vol. 11. 2014.
E.C. Howard et al: "Finite Elements and Fast Iterative Solvers: with applications in Incompressible Fluid Dynamics", $2^{\text {nd }}$ Edition, Oxford Press, UK. 2014.

M.O. Oyesanya. "Duffing Oscillator as a model for predicting earthquake occurrence". I. J of NAMP. 12:133-142, 2008

J. Sunday: "The Duffing Oscillator; Applications and Computational Simulations", Asian Research Journal of Mathematics, DOI: 10.9734/ARJOM/2017/31199

I. Kovacic, M. J. Brennan:" The Duffing Equation; Nonlinear Oscillators and their Behaviours", 1st Edition, John Wiley and Sons Publishers, UK. 2011.

V. Marinca; N. Herisanu: "Explicit and Exact Solutions to Cubic Duffing and Double - Well Duffing Equations". Mathematical and Computer Modelling Journal, 53., 2011 604/609; Elsevier Publications.

Y. Guo, A. C. J. Ruo: "Periodic Motions in a Double - Well Duffing Oscillator under Periodic Excitation Through Discrete Implicit Mappings". Int'l Journal of Dynamics and Control; ISSN 2195 268X, DOI: 10.1007/s40435-015-0161-6. Springer, 2015.

J. M. Thompson: "Basin Explosions and Escape Phenomena in the Twin - Well Duffing Oscillator: Compound Global Bifurcations Organizing Behaviour". Philosophical Transition of the Royal Society London. Research Gate Publications. 332, DOI: 10.1098/rsta. 1990.

M. Zukovic, I. Kovacic, M. P. Cartmell: "Characterising the Dynamic Behaviour of Two-Well Oscillators Excited at Low Frequency: Numerical Insights". Journal of the Serbian Society for Computational Mechanics: Vol. 91. No. 2. 2015/pp. 34-36; UDC: 621.373.029.4.015.53.

O. C. Zienkiewicz; R. L. Taylor; J. Z. Zhu: The Finite Element Method; its basis and fundamentals. 7th Edition, Butterworth-Heinemann Publishers, UK. 2013.

I. Koutromanos: "Fundamentals of Finite Element Analysis", Wiley Pub., Canada, 2018. 